\title{
Automatic Extraction of Probabilistic Workload Specifications for Load Testing Session-Based Application Systems
}

\author{
André van Hoorn, ${ }^{1}$ Christian Vögele, ${ }^{2}$ Eike Schulz, ${ }^{3}$ \\ Wilhelm Hasselbring, ${ }^{3}$ Helmut $\mathrm{Krcmar}^{4}$ \\ ${ }^{1}$ University of Stuttgart, Institute of Software Technology, 70569 Stuttgart, Germany \\ ${ }_{2}$ fortiss $\mathrm{GmbH}, 80805$ München, Germany \\ ${ }^{3}$ Kiel University, Department of Computer Science, 24118 Kiel, Germany \\ ${ }^{4}$ Technische Universität München, Chair for Information Systems, 85748 Garching, Germany \\ andre.van.hoorn@acm.org, voegele@fortiss.org, \{esc,wha\}@informatik.uni-kiel.de,krcmar@in.tum.de
}

\begin{abstract}
Workload generation is essential to systematically evaluate performance properties of application systems under controlled conditions, e.g., in load tests or benchmarks. The definition of workload specifications that represent the real workload as accurately as possible is one of the biggest challenges in this area. This paper presents our approach for the modeling and automatic extraction of probabilistic workload specifications for load testing session-based application systems. The approach, called WessBas, comprises (i.) a domain-specific language (DSL) enabling layered modeling of workload specifications as well as support for (ii.) automatically extracting instances of the DSL from recorded sessions logs and (iii.) transforming instances of the DSL to workload specifications of existing load testing tools. During the extraction process, different groups of customers with similar navigational patterns are identified using clustering techniques. We developed corresponding tool support including a transformation to probabilistic test scripts for the Apache JMeter load testing tool. The evaluation of the proposed approach using the industry standard benchmark SPECjEnterprise2010 demonstrates its applicability and the representativeness of the extracted workloads.
\end{abstract}

\section{Categories and Subject Descriptors}

C.4 [Performance of Systems]: measurement techniques, modeling techniques

\section{Keywords}

Workload Specifications, Load Testing, Clustering, Sessionbased Application Systems, Load Test Extraction

\section{INTRODUCTION}

For essentially any measurement-based software performance evaluation activity-e.g., load, stress, and regression testing, or benchmarking - it is necessary to expose the system

Permission to make digital or hard copies of all or part of this work for personal or classroom use is granted without fee provided that copies are not made or distributed for profit or commercial advantage and that copies bear this notice and the full citation on the first page. To copy otherwise, to republish, to post on servers or to redistribute to lists, requires prior specific permission and/or a fee.

VALUETOOLS 2014, December 09-11, Bratislava, Slovakia

Copyright @ 2015 ICST 978-1-63190-057-0

DOI 10.4108/icst.valuetools.2014.258171 under test (SUT) to synthetic workload [3, 5, 6, 7], i.e., automatically generating requests to system-provided services. Workload generation tools - also called workload driversare used to emulate a multitude of concurrent system users based on workload specifications, ranging from manually defined scenarios over recorded traces to analytical models [1]. This paper focuses on analytical model-based workload generation for session-based systems, i.e., systems that are used by users in time-bounded sessions of interrelated requests and think times between subsequent requests [8].

Approaches have been proposed for specifying and generating workloads for this type of systems (e.g., $[6,7,8]$ ). However, one of the biggest challenges is how to obtain workload specifications that produce workload characteristics similar to a system's production usage profile, e.g., arrival rates of sessions and requests to system-provided services. Further, the extraction and specification of workloads strongly depends on the used workload generation tool. Because of that the workload must be extracted for each tool and specified into a specific structure.

In response to these challenges, this paper presents our WESS$\mathrm{BAS}^{1}$ approach for specifying and extracting probabilistic workloads for session-based application systems. A domainspecific language (DSL), called WESSBAS-DSL, is introduced which enables the system- and tool-agnostic modeling of these workload specifications. Recorded session logs from the systems are used as basis for the automatic extraction of Wessbas-DSL instances. Different groups of customers showing similar navigational patterns are identified during the creation of these instances. WESSBAS-DSL instances are transformed to workload specifications for load generation tools. Finally, a transformation to the common load testing tool Apache JMeter, including the Markov4JMeter extension developed in our previous work [14], is presented in this paper. Figure 1 provides an overview of the WeSSBAS approach.

To summarize, the contribution of this paper is our WESSBAS approach for automatic extraction of probabilistic workload specifications of session-based application systems, comprising (i.) a DSL for modeling session-based probabilistic workload specifications, (ii.) an automatic extraction of DSL

${ }^{1}$ WESSBAS is an acronym for Workload Extraction and Specification for Session-Based Application Systems 


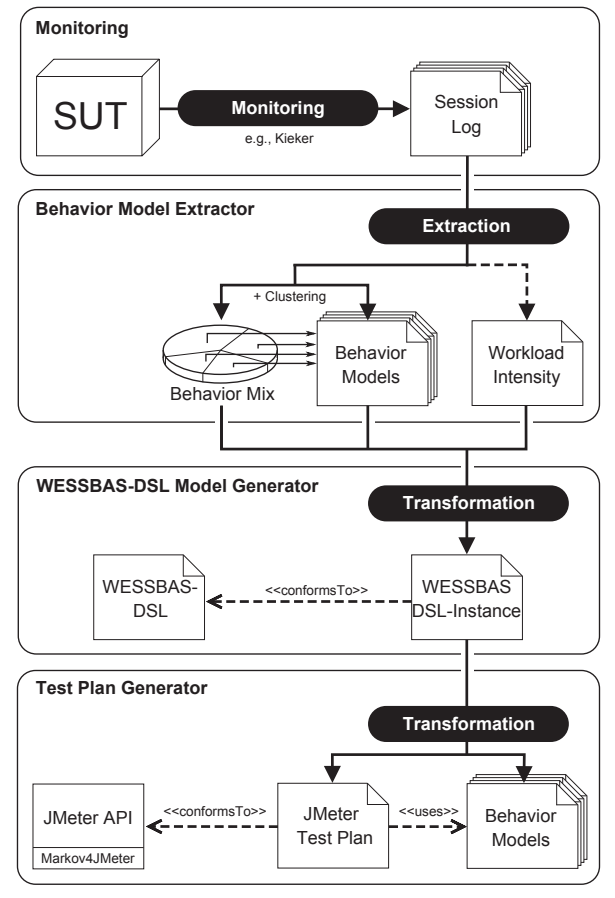

Figure 1: Overview of the WESSBAS approach

instances from recorded sessions logs including the clustering of navigational patterns, (iii.) transformations from DSL instances to JMeter Test Plans, and (iv.) tool support for this approach. The tool support serves as an extensible implementation of the approach, including the DSL, the extraction, as well as a proof-of-concept transformation from the DSL to JMeter Test Plans. Supplementary material for this paper, including the developed tools, models, and experimental results, is publicly available online. ${ }^{2}$

\section{BACKGROUND AND RELATED WORK}

The approach described in this paper builds on our previous work on generating probabilistic and intensity-varying workloads [11, 14] for session-based systems - particularly the workload modeling formalism that extends the work by Menascé et al. [8] and Krishnamurthy et al. [6]. This section introduces the concepts needed for the remainder of this paper, including a brief discussion of related work.

The workload modeling formalism (Workload Model) comprises two different types of models, which will be detailed below [14]: (i.) an Application Model, specifying allowed sequences of service invocations and SUT-specific details for generating valid requests; and (ii.) a weighted set of Behavior Models, each providing a probabilistic representation of user sessions in terms of invoked services and think times among subsequent invocations. Additionally, the Workload Model includes a function specifying the number of active sessions during the workload generation execution. We developed a publicly available extension, called Markov4JMeter [14], for the well-known load generator Apache JMeter, allowing to define and execute these Workload Models.

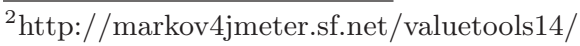

The Application Model is a two-layered hierarchical finite state machine (FSM), consisting of a Session Layer and a Protocol Layer. Inspired by the work by Krishnamurthy et al. [6], the Session Layer is a finite state machine, in which states refer to system-provided services and allowed transitions among these-possibly labeled with guards and actions. A guard is a boolean expression, defining the condition under which the related application transition fires. An action is a list of statements to be executed, in case the related application transition fires. For each Session Layer state, the Protocol Layer contains an associated FSM, which models the sequence of protocol-level requests to be executed when the Session Layer state is executed. The transitions define the valid sequences from one state to another.

A Behavior Model roughly corresponds to the Customer Behavior Model Graphs (CBMGs) introduced by Menascé et al. [8]. A Behavior Model $\mathcal{B}_{A}$ is defined as a tuple $\left(S \cup\{\$\}, P, z_{0}, f_{t t}\right)$. $S$ specifies the set of states contained in the Behavior Model with initial state $z_{0} \in S$ and exit state $\$ . P=\left[p_{i, j}\right]$ is an $n \times n$-matrix of transition probabilities, with $n=|S \cup\{\$\}|$. A matrix entry $p_{i, j}$ defines the probability for a transition from state $i$ to state $j$. The distribution function $f_{t t}$ specifies the think time associated with a transition. Think times can, for instance, be modeled by using random values. The Behavior Mix is a set $\left\{\left(\mathcal{B}_{0}, r_{0}\right), \ldots,\left(\mathcal{B}_{m-1}, r_{m-1}\right)\right\}$, which assigns a relative frequency $r_{i}$ to the Behavior Model $\mathcal{B}_{i}$. A tuple $\left(\mathcal{B}_{i}, r_{i}\right)$ indicates that sessions that correspond to the Behavior Model $\mathcal{B}_{i}$ are generated with a relative frequency of $r_{i} \in[0,1]$. During the workload generation process to a SUT, the Behavior Mix determines the user type to be emulated next by selecting the corresponding Behavior Model based on the assigned relative frequencies.

In the proposed approach, workload specifications representing the measured usage profiles of session-based systems are extracted. A similar approach, yet focusing on the aforementioned CBMGs, has been proposed by Menascé et al. [7, 8]. The authors extract CBMGs from HTTP server logs, including K-means clustering to identify CMBGs for similar types of users. In contrast, in our work an advancement of the K-means algorithm, called X-means is applied.

Our approach focuses on the specification of the behavior of users and offers basic support for modeling workload intensities. An approach focusing on the definition of the workload intensities can be found in [13]. It allows a DSL-based definition of variable and dynamic load profiles and workload scenarios over time.

\section{WESSBAS-DSL}

The Wessbas domain-specific language (DSL), referred to as WESSBAS-DSL, follows the Markov4JMeter workload modeling formalism [14] introduced in the previous section and therewith denotes a language for expressing such models. In our approach, the WESSBAS-DSL is used as an intermediate language between the construction of SUT-specific but tool-agnostic workload models on the one side, and the generation of corresponding inputs to load testing tools on the other side. The WESSBAS-DSL is implemented as an Ecore-based meta-model, using the benefits and tool support 


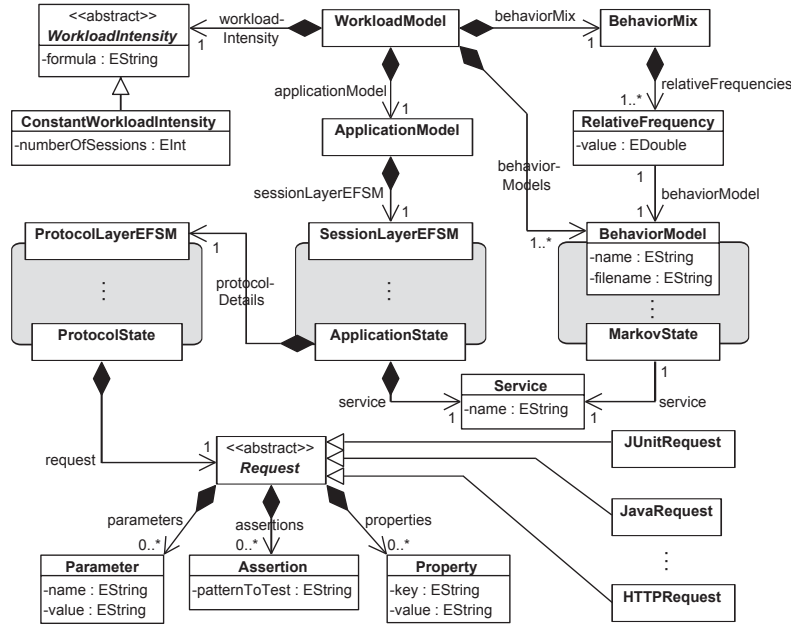

(a) Wessbas-DSL classes and relationships

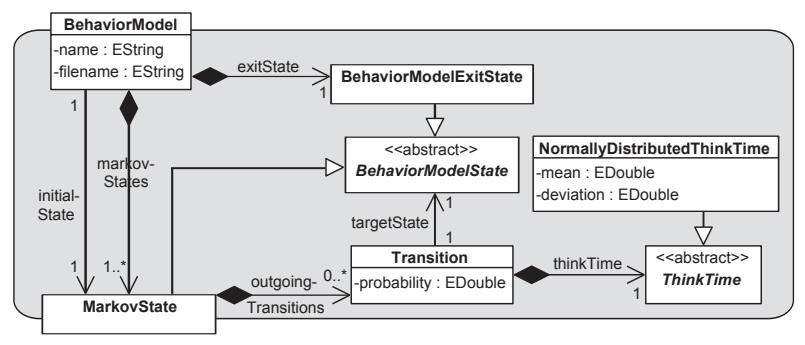

(b) Details on the Behavior Model

Figure 2: Overview of the WessBas-DSL

of the widely spread Eclipse Modeling Framework (EMF). ${ }^{3}$ The meta-model is enriched with a comprehensive amount of constraints (specified in the common Object Constraint Language, OCL), for checking the validity of model instances. The WessBAs-DSL structure offers a high degree of flexibility and extensibility. The remainder of this section introduces the core concepts of the Wessbas-DSL. Details are also provided by Schulz [10].

As a language for the Markov4JMeter workload modeling formalism, the WESSBAS-DSL includes the essential components of that model, in particular the Application Model, the (Behavior) Mix of Behavior Models, and the workload intensity, as introduced in Section 2. Figure 2a gives an overview of the WESSBAS-DSL class structure.

The representation of the Application Model corresponds to the two-layered structure of that component, including FSMs for the Session Layer and the Protocol Layer as well. States of the Session Layer FSM, shortly referred to as Application States, are associated with services and Protocol Layer FSMs. States of the Protocol Layer FSMs are associated with protocol-specific requests, which might be of type HTTP, Java, JUnit, BeanShell, SOAP, etc.; the set of currently supported request types can be extended easily by deriving additional subclasses from the common base class. Mention should be made of the difference between properties

$\overline{{ }^{3} \text { http://www.eclipse.org/modeling/emf/ }}$ and parameters of a request: properties correspond to the information which is required for sending a request, e.g., domain, path, or port number of a targeted server; parameters denote values to be sent with the request, e.g., input data for a web form. Behavior Models are modeled as FSMs, with (Markov) States being associated with services. Figure 2b illustrates the class structure of Behavior Models. Transitions are labeled with probabilities and think times, whereas think times follow a certain type. Currently supported think times are of type Gaussian, that is, they underlie a normal distribution, indicating mean and (standard) deviation values as parameters. Exit states are modeled explicitly, as they arein contrast to Markov States - not associated with services. Each Behavior Model is associated with a relative frequency, stored as a double value in a dedicated class. These frequencies are contained in the Behavior Mix, whose corresponding class denotes a further component of the workload model. Session Layer and Protocol Layer FSMs are modeled analogous to Behavior Models, with transitions being labeled with guards and actions. The formula for the workload intensity is stored as a string attribute in a dedicated class that also serves as a base class for all types of workload intensity. This facilitates a simple installation of according formulas, which might be provided by appropriate tools (e.g., [13]).

Even though the WessBAS-DSL is independent of specific testing tools, it includes all core information required for generating workload specifications that build on the Markov4JMeter workload modeling formalism. In this paper, we exemplify this by generating JMeter Test Plans through passing WeSSBAS-DSL models as input to a transformation tool. This will be further discussed in Section 5. The implementation of the WESSBAS-DSL as an Ecore meta-model offers the benefits of EMF tools such as EMF Form Editors or serialization support. In particular, WESSBAS-DSL instances can be viewed, validated, and modified in an editor, before being passed as input to any transformation process. The EMF Form Editor offers a constraint Live-Validation option, which facilitates the maintenance of Wessbas-DSL models. The extensibility of the WessBasDSL is given through its class structure: additional types of workload intensity, requests, or think times can be simply implemented by deriving appropriate subclasses from the related base classes.

\section{EXTRACTING WESSBAS-DSL INSTANCES}

The extraction of WESSBAS-DSL instances is based on socalled session logs obtained from raw session information, recorded from a running application system. Raw session information is usually provided by a request log generated by monitoring facilities, comprising the associated requests to system-provided services with a session identifier and timestamps for the request and completion time. A typical example is the HTTP request log provided by common web servers [8], or tracing information obtained from applicationlevel monitoring tools [15]. The session log groups the requests by the session identifier, giving access to the sequence and timing information of subsequent service requests within a session. We will not detail the process of obtaining session logs from request logs any further but refer to existing works [8] and assume that a session log in the WessBAs format is available. The remainder of this section details the twostep procedure to obtain a WESSBAS-DSL instance, compris- 
ing the (i.) clustering-based extraction of the Behavior Mix (Section 4.1), and the (ii.) generation of a complete WessBAS-DSL instance from the Behavior Mix (Section 4.2).

\subsection{Clustering-Based Behavior Mix Extraction}

During the transformation of a session log to a WESSBASDSL instance, the Behavior Mix is determined by identifying different groups of customers with similar navigational patterns. As proposed in [8], clustering methods can be used to support this task. The identification of different customer groups has several advantages. First, the system can be optimized upon these navigational patterns. Further, the impact of different Behavior Mixes on the performance can be evaluated, e.g., investigating the performance impact of an increased fraction of heavy buyers. To reduce the complexity and to increase the comprehensibility of the resulting Behavior Mix, the goal of the clustering is to obtain a relative small number of clusters.

In this paper, we focus on clustering with the centroid-based $\mathrm{X}$-means algorithm, which is an advancement of the wellknown K-means algorithm [9]. The advantage of X-means over K-means is, that it is not mandatory to specify the number of clusters $\mathrm{K}$ in advance by the user. The user provides a minimum and a maximum number of resulting clusters and the algorithm determines how many clusters are best suited. The evaluation of K-means clustering is very costly as the results of the K-means must repeatedly be evaluated with different numbers of K [2]. Further, the X-means algorithm scales better and the risk of finding local minima is lower. The X-means clustering algorithm is integrated into our proposed approach using the data mining framework Weka [4]. Other algorithms can be integrated accordingly.

Input instances for the clustering are absolute Behavior Models, each representing a $n \times n$-matrix of absolute transition frequencies of one user session. Think times are not part of the clustering as they have no impact on the navigational patterns. Each matrix is transformed into a vector, as Weka cannot handle matrices as clustering input. Therefore, the values of a matrix is transformed into a vector by concatenating the rows of the matrix. In a first step, a central vector, called centroid, is determined randomly for each cluster. Each centroid represents a cluster and is the mean of the instances in that cluster. Then, the clustering algorithm iterates several times over the dataset and assigns instances to the nearest cluster centroid, until no instance changes the cluster anymore.

The distance between the instances is calculated using the Euclidean distance metric. During the calculation of a distance, the attributes of the instances can be normalized to a value between zero and one. Without data normalization, attributes with highest variance are driving the clustering. That means in our case, that high transition counts have a high influence on the clustering. In order to figure out the best settings, both the normalized and the non-normalized Euclidean distance will be evaluated in Section 6.3.

Having executed the clustering, each attribute of a centroid represents the mean of the respective attribute values of all instances within this cluster. As a result, the centroids represent the absolute Behavior Model of the corresponding cluster. Think times per cluster centroid are determined by calculating the sum of the think times per transition of the respective cluster instances. Finally, the resulting Behavior Mix is calculated like proposed in [8]. It consists of the (relative) Behavior Models, the mean think times per transition and the relative frequencies of the Behavior Models.

\subsection{Generating WESSBAS-DSL Instances}

The next task is to transform the extracted Behavior Models and the determined Behavior Mix to a valid WessBAsDSL instance, which can be further transformed to any test script format. Therefore, our dedicated Java-based implementation, namely Wessbas-DSL Model Generator (Figure 1), performs the following three steps: (i.) construction of an Application Model, based on SUT-specific states and transitions, (ii.) integration of the determined Behavior Mix including the extracted Behavior Models, and (iii.) integration of the workload intensity definition.

The construction of an Application Model builds on SUTspecific information, particularly validness of service execution sequences for the Session Layer FSM and protocolspecific information for the Protocol Layer FSMs. The range of such information differs as well as the format it might be provided in; consequently, extensions might be necessary. In our approach, the Behavior Model Extractor tool (Figure 1) outputs a list of all available services associated with any states of Behavior Models. (Note that SUT-specific Behavior Models are defined on a common set of services.) A small script converts this list into a format that can be processed by the Wessbas-DSL Model Generator. This information can be enriched with transition specifications; currently, our script generates all possible transitions between services, assuming all sequences of service executions are valid. In particular, neither transition guards nor actions are considered.

After reading the appropriately-formatted input data, the Wessbas-DSL Model Generator builds a corresponding Session Layer FSM and assigns Protocol Layer FSMs to the Markov States. Assuming that a (virtual) user provides valid input only, the structure of our Protocol Layer FSMs remains trivial with exactly one Protocol State per FSM, indicating exactly one request being sent in a Markov State. A DSL that allows the definition of more complex, protocolspecific FSMs, e.g., failed user logins, denotes a future work issue.

The integration of Behavior Mix and Behavior Models includes the construction of corresponding WESSBAS-DSL fragments. As the Application Layer includes all available services, corresponding Behavior Models can be derived, to be equipped with probabilities and think times provided by the extracted Behavior Models. Finally, the workload intensity is read as a formula string from a properties file, to be included into the resulting model. For further processing, the resulting WESSBAS-DSL model is serialized to an XMI file, using dedicated Ecore techniques. That file can be loaded into an EMF Form Editor to be validated and analyzed, before being passed to the next transformation module.

\section{GENERATING JMETER TEST PLANS}

The final task of the extraction process is to transform a given WessBAS-DSL instance into a corresponding JMeter 
Table 1: Mapping of Wessbas-DSL concepts to (Markov4)JMeter elements

\begin{tabular}{l|l}
\hline WESSBAS-DSL & Markov4JMeter Elements \\
\hline \hline Session Layer FSM & Markov States (+ outgoing transitions) \\
Protocol Layer FSMs & JMeter Elements (Markov State children) \\
Workload Intensity & MSC (Session Arrival Controller) \\
Behavior Mix & MSC (frequency table) \\
\hline Behavior Models & MSC (frequency table) $\rightarrow$ CSV-files \\
\hline
\end{tabular}

Test Plan. Our Java-based implementation, namely Test Plan Generator (Figure 1), reads a serialized WessBAS-DSL instance from file and constructs a further XMI structure, which can be processed by the JMeter tool. The XMI output is generated via the JMeter API and denotes a JMetertypical tree structure of Test Plan elements, including Markov4JMeter-specific elements, namely Markov States and Markov Session Controller, that are provided by the Markov4JMeter add-on for JMeter. The core transformation process builds on a mapping between WessBAS-DSL concepts and (Markov4)JMeter Test Plan elements. An overview of the underlying mappings is given in Table 1.

A Session Layer FSM in the Wessbas-DSL is mapped to a corresponding set of Markov States in JMeter. Each Markov State includes its individual set of outgoing transitions with guards and actions, for defining the validity of state execution sequences. The name of a Markov State in the resulting JMeter Test Plan corresponds to the name of the state's associated service in the WessBas-DSL instance. Protocol Layer FSMs are modeled as child elements of Markov States in the tree-structured result. They are constructed with the use of JMeter controllers and samplers as well, according to their related WESSBAS-DSL structure. The workload intensity is stored as a formula string in the Session Arrival Controller sub-component of a Test Plan's (unique) Markov Session Controller. That controller additionally includes a table for Behavior Mix frequencies, to be filled with according values of the input WESSBAS-DSL instance. Behavior Models are stored separately - indicated by a separation line in Table 1-in CSV-files, which are referred by the frequency table of the Markov Session Controller.

Besides the Test Plan elements that result from the core transformation process for a given WESSBAS-DSL instance, several JMeter elements are added to a generated Test Plan by default. This step is required for making a Test Plan's structure accessible for the JMeter tool and providing additional functionality, such as handling of HTTP session cookies. Currently, the Test Plan structure is predefined, targeting HTTP-based tests only; an appropriate mechanism for specifying alternative structures, particularly for different types of requests, denotes a future work issue.

\section{EVALUATION}

In this evaluation, we apply our proposed extraction approach and tooling to the industry-standard benchmark SPECjEnterprise2010. This serves as an investigation of (i.) the practicality of the approach and tooling support and (ii.) the representativeness of the extracted workload specifications. With respect to (ii.) we particularly investigate the following two research questions: (RQ 1) How accurately do the clustering results match the input Behavior Mix? and $(R Q$ 2) What is the impact of the clustering results on the workload characteristics? Section 6.1 describes the experimental setting. The SPECjEnterprise 2010 deployment is explained in Section 6.2. The results for RQ 1 and RQ 2 are detailed in Sections 6.3 and 6.4.

\subsection{Evaluation Methodology}

An instrumented version of SPECjEnterprise $2010^{4}$ is executed with three different Behavior Mixes to obtain a session log, from which instances of the WESSBAS-DSL are extracted and transformed into JMeter Test Plans. For the Behavior Model extraction we applied different configurations of the X-means clustering. A basic Application Model is automatically generated from the obtained Behavior Models. Its Session Layer comprises the superset of all states from the Behavior Models, assuming that all transitions between all states are allowed (no guards and actions). The Protocol Layer comprises a mockup HTTP request per state. The transformation from the instances to JMeter Test Plans is performed according to Section 4. In order to measure the characteristics of extracted workload models, we developed a web application that is instrumented according to the SPECjEnterprise2010. Hence, the same session log analysis infrastructure can be applied to both the session information obtained from the SPECjEnterprise2010 runs and the JMeter runs for the synthetic workloads of the extracted workload specifications. The reason why we do not execute the extracted workload against the SPECjEnterprise2010 is that currently input parameters for the workload are not extracted automatically.

The accuracy of the clustering (RQ 1) is evaluated based on the fraction of misclassified sessions over all classifications of the clustering for a benchmark run. The impact of the clustering on the workload characteristics (RQ 2) is evaluated based on (i.) two session-based metrics, session length as number of requests per sessions and number of distinct session types, as well as (ii.) a request-based metric, namely the relative invocation frequency of all request types. Note that due to the nature of the SPECjEnterprise2010 workload we do not consider timing-related metrics such as think times or arrival rates, even though they are correctly extracted and executed by our approach. Conclusions about the arrival rates of requests can be drawn by looking at the invocation frequencies of requests.

\subsection{SPECjEnterprise2010 Deployment}

SPECjEnterprise2010 is a Java EE application representing a business case that combines customer relationship management (CRM), supply chain management (SCM), and manufacturing. It includes a workload specification and a dataset needed for the execution of load tests. The workload is generated by the Faban Harness and Benchmark Driver. ${ }^{5}$ The benchmark consists of three different application domains, namely Orders domain (CRM), Manufacturing domain, and

\footnotetext{
${ }^{4}$ SPECjEnterprise is a trademark of the Standard Performance Evaluation Corp. (SPEC). The SPECjEnterprise2010 results or findings in this publication have not been reviewed or accepted by SPEC, therefore no comparison nor performance inference can be made against any published SPEC result. The official web site for SPECjEnterprise2010 is located at http://www.spec.org/jEnterprise 2010 .

${ }^{5}$ http://java.net/projects/faban/
} 


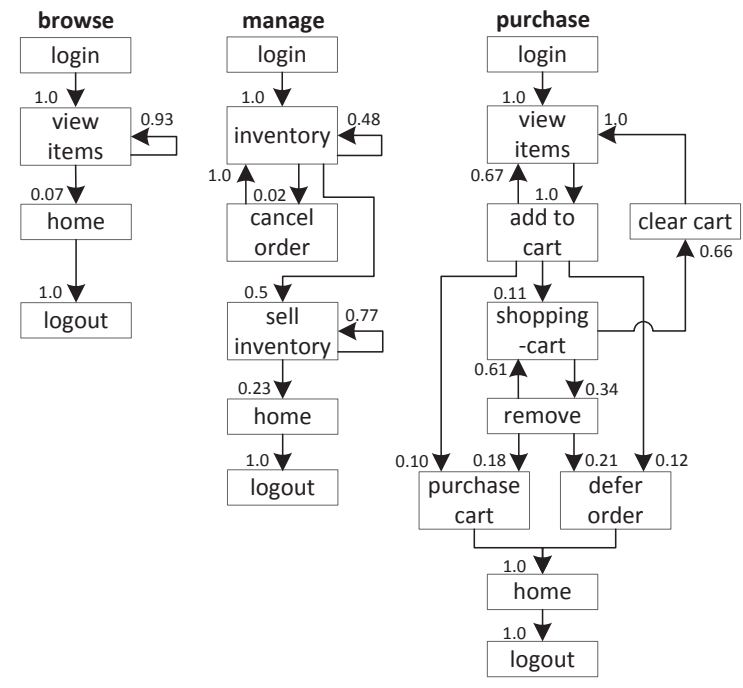

Figure 3: SPECjEnterprise2010 transactions as Behavior Models

Supplier domain (SCM). In this work we consider only the Orders domain, which represents a typical web-based application that provides e-commerce functionality to the customers - in this case automobile dealers. The customers are able to purchase and sell cars, to manage their accounts and dealership inventory, and to browse the catalogue of cars. The Orders domain represents the SUT.

\subsubsection{Workload Description}

SPECjEnterprise2010 defines three different transaction types which are executed by automobile dealers: Browse (B), Manage (M), and Purchase (P). Within Browse, the benchmark driver navigates to the catalogue of available cars and browses the catalogue for a constant number of times. Manage describes a scenario where open orders are canceled and vehicles are sold. In the more complex transaction type Purchase, orders are placed and immediately purchased or deferred. The shopping cart is either cleared or items are removed one by one until only one item remains. Each of these transaction types is a sequence of HTTP requests. In total, 13 different HTTP request types are defined. Within the transactions, no think times are defined, i.e., each HTTP action is executed directly after its previous request has been completed. Therefore, the evaluation of think times extraction is out of scope for this paper. Figure 3 depicts the structure of the three transaction types as Behavior Models obtained by applying our WESSBAS extraction approach.

In the original benchmark workload, automobile dealers log in to the system, execute multiple instances of the three transactions types, and $\log$ out. Each of the three transaction types is executed with a specified probability. The standard transaction mix is 50\% Browse, 25\% Manage, and $25 \%$ Purchase. We modified the dealer driver such that each transaction starts with a login and ends with a logout. This way each transaction corresponds to a unique session, and the transaction mix corresponds to the Behavior Mix.

\subsubsection{Benchmark Execution and Monitoring}

Three different transaction mixes are used to evaluate the proposed approach. For each mix, one of the transaction types is executed with a probability of $50 \%$ and the other two with $25 \%$ each. A load of 800 concurrent users is executed, resulting in a moderate CPU utilization of the SUT of approximately $40 \%$. Each benchmark run is executed for ten minutes after a four minute ramp-up phase and before a four minute ramp-down phase.

In order to obtain the raw session information, the SUT was instrumented using Kieker [15]. For each request the requested URL, the query string, the session ID, and the server-side entry and exit timestamp are recorded. Afterwards, the raw log data is transformed to a session log. During the transformation, the sessions within the ramp-up and ramp-down phase are not taken into account. In order to be able to evaluate the clustering results of the transaction types, the name of the transaction type is added as additional parameter to the login HTTP action.

\subsection{Accuracy of Clustering}

The evaluation of clustering accuracy (RQ 1) is split into two steps. In the first step, the accuracy of the clustering is determined based on the assumption that the number of resulting clusters is known in advance. For this reason, the number of resulting clusters is fixed to three. As the number of clusters is usually not known in advance, we let the $\mathrm{X}$-means algorithm determine the number of clusters in a second step. As the seed value for the random selection of the initial centroids can have a high impact on the clustering results, multiple clustering runs with different seed values between one and twelve are executed. Afterwards, the run with the lowest sum of squared error value [9] is selected.

The results of the clustering are presented in Table 2. For each transaction mix (TM), the clustering shows for each transaction type $(\mathrm{T})$ the cluster $\left(\mathrm{C}_{x}\right)$ where a transaction is assigned to, and the percentage of misclassified (MC) transactions. The left side shows the results of exactly three predefined clusters (step one); the right side shows the results letting X-means determine the number of clusters between two and twenty (step 2). The number of transactions (N) clustered for each transaction mix is around 61,000 .

The results using exactly three clusters indicate that the clustering using normalized Euclidean distance (NED) is able to cluster all transactions correctly (100\%) resulting in the Behavior Models shown in Figure 3. The clustering using Euclidean distance (ED) without normalization classifies the transactions Browse and Manage correctly, whereas a fraction of transactions of type Purchase is assigned mistakenly to the same cluster as the Manage transactions. In the second transaction mix, the fraction of Purchase transactions is higher than in the other mixes. Hence, the percentage of misclassified transactions is with $15.98 \%$ relatively high.

The clustering without predefining the correct number of clusters, results in two clusters using ED and four clusters using NED. As clustering with ED always merges transactions of type Purchase and Manage, the percentage of misclassified transactions is around $25 \%$ for all mixes. It is assumed that the transaction type with the lower number of 
Table 2: Clustering Results

\begin{tabular}{|c|c|c|c|c|c|c|c|c|c|c|c|c|c|c|c|c|c|c|}
\hline & \multicolumn{8}{|c|}{$\mathrm{X}$-means (min 3 cluster, $\max 3$ cluster) } & \multicolumn{8}{|c|}{ X-means (min 2 cluster, max 20 cluster) } & \multirow{3}{*}{$\mathrm{N}$} \\
\hline & & \multicolumn{4}{|c|}{ ED } & \multicolumn{4}{|c|}{ NED } & \multicolumn{3}{|c|}{ ED } & \multicolumn{5}{|c|}{ NED } & \\
\hline TM & $\mathrm{T}$ & C1 & $\mathrm{C} 2$ & C3 & MC & C1 & $\mathrm{C} 2$ & C3 & MC & C1 & $\mathrm{C} 2$ & $\mathrm{MC}$ & C1 & $\mathrm{C} 2$ & $\mathrm{C} 3$ & $\mathrm{C} 4$ & $\mathrm{MC}$ & \\
\hline 50 & B & 0 & 0 & 31,060 & \multirow{3}{*}{$2.91 \%$} & 0 & 31,060 & 0 & \multirow{3}{*}{$0 \%$} & 0 & 31,060 & \multirow{3}{*}{$24.62 \%$} & 0 & 0 & 0 & 31,060 & \multirow{3}{*}{$1.03 \%$} & \multirow{3}{*}{61,500} \\
\hline 25 & M & 15,298 & 0 & 0 & & 15,298 & 0 & 0 & & 15,298 & 0 & & 632 & 14,666 & 0 & 0 & & \\
\hline 25 & $\mathrm{P}$ & 1,789 & 13,353 & 0 & & 0 & 0 & 15,142 & & 15,142 & 0 & & 0 & 0 & 15,142 & 0 & & \\
\hline 25 & $\mathrm{~B}$ & 15,091 & 0 & 0 & \multirow{3}{*}{$15.98 \%$} & 15,091 & 0 & 0 & \multirow{3}{*}{$0 \%$} & 0 & 15,091 & \multirow{3}{*}{$24.96 \%$} & 0 & 15,091 & 0 & 0 & \multirow{3}{*}{$15.30 \%$} & \multirow{3}{*}{60,089} \\
\hline 25 & $\mathrm{M}$ & 0 & 0 & 15,000 & & 0 & 15,000 & 0 & & 15,000 & 0 & & 0 & 0 & 707 & 14,293 & & \\
\hline 50 & $\mathrm{P}$ & 0 & 20,397 & 9,601 & & 0 & 0 & 29,998 & & 29,998 & 0 & & 21,513 & 8,485 & 0 & 0 & & \\
\hline 25 & B & 0 & 15,231 & 0 & \multirow{3}{*}{$2.99 \%$} & 15,231 & 0 & 0 & \multirow{3}{*}{$0 \%$} & 0 & 15,231 & \multirow{3}{*}{$25.16 \%$} & 0 & 0 & 0 & 15,231 & \multirow{3}{*}{$1.86 \%$} & \multirow{3}{*}{61,118} \\
\hline 50 & M & 30,510 & 0 & 0 & & 0 & 30,510 & 0 & & 30,510 & 0 & & 29,375 & 1,135 & 0 & 0 & & \\
\hline 25 & $\mathrm{P}$ & 1,824 & 0 & 13,553 & & 0 & 0 & 15,377 & & 15,377 & 0 & & 0 & 0 & 15,377 & 0 & & \\
\hline
\end{tabular}

instances merged within one cluster count as missclassified. The clustering using NED always correctly classifies Browse transactions. Manage transactions are always split into two clusters whereas Purchase is only split into two clusters in the second transaction mix. Hence, the percentage of misclassified transactions is again relatively high (15.3\%) in the second transaction mix.

Transactions of type Browse seem to be homogeneous in a way that they were clustered correctly among all clustering runs. This can be explained as Browse transactions are executed with a constant number of actions without probabilistic behavior. NED is better suited to cluster the different transaction types than the non-normalized version. The normalization has the effect that high transaction counts and therefore also the length of the sessions has a lower impact on the clustering. Thus, the structure of the transactions in terms of the number of different HTTP requests grows in significance. As each of the three transaction types consist of different HTTP request types (except for login, home and logout), the clustering results are significantly better.

\subsection{Accuracy of Workload Characteristics}

To evaluate the accuracy of the extracted workload specifications (RQ 2), we compare the server-side session-based and request-based metrics mentioned in Section 6.1 for the original measurements with the corresponding metrics obtained by executing extracted workload specifications using JMeter. Due to space limitations, we present only the results of the original benchmark Behavior Mix (25\% P, 50\% $\mathrm{B}$, and $25 \% \mathrm{M}$ ), using the X-means clustering algorithms results with 2 (ED), 3 (NED), and 4 (NED) clusters (entries for the bottom TM in Table 2). The original workload includes 61,500 sessions and 847,927 HTTP requests. These numbers served as an approximate stopping criteria during the execution of the synthetic workload with JMeter (cf. Figure $4 \mathrm{~b}$ and Figure 5b).

\subsubsection{Session Length and Distinct Sessions}

Statistics about the session length distributions of the original and the three synthetic workloads are listed in Figure 4. Looking only at the mean values and the 0.95 confidence interval (Figure 4b), one may conclude that the session length distributions of the three synthetic workloads exactly match the distribution of the original workload. However, particularly the violin plot (Figure 4a) indicates that the synthetic distributions are similar but differ considerably from

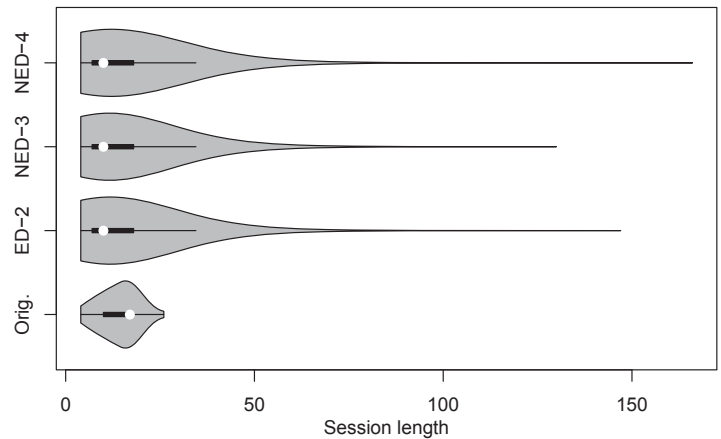

(a) Violin plot (combination of box and density plot)

\begin{tabular}{l||r|r|r|r|r|r|r|c}
\hline & Min. & $Q_{1}$ & Med. & Mean & CI $_{0.95}$ & $Q_{3}$ & Max. & $N$ \\
\hline \hline Orig. & 4 & 10 & 17 & 14.23 & {$[14.19,14.26]$} & 17 & 26 & 61,500 \\
\hline ED-2 & 4 & 7 & 10 & 14.24 & {$[14.15,14.33]$} & 18 & 147 & 60,957 \\
\hline NED-3 & 4 & 7 & 10 & 14.24 & {$[14.15,14.33]$} & 18 & 130 & 62,054 \\
\hline NED-4 & 4 & 7 & 10 & 14.26 & {$[14.17,14.35]$} & 18 & 166 & 59,971 \\
\hline \hline
\end{tabular}

(b) Summary statistics

Figure 4: Session length statistics for the original workload (Orig.) and the synthetic workloads (ED-2, NED-3, NED-4)

the original workload. The quartile-based statistics in Table $4 \mathrm{~b}$ confirm this observation. It can be observed that for the synthetic workloads, very long sessions are generated. While for the original workload the longest sessions comprise 26 requests, the synthetic sessions reach maximums of 147, 130, and 166. Looking at the individual session lengths, $11 \%$ of the synthetic sessions are longer than the longest sessions of the original workload.

In the original workload, we identified 78 distinct sessions. The number of distinct sessions in the synthetic workloads is considerably higher, namely 2, 126 (2 clusters), 2, 144 (3 clusters), 1, 996 (4 clusters). The relatively low number of distinct session types is caused by the fact that the original SPECjEnterprise2010 workload contains only few probabilistic elements, which are all bounded in the number of maximum iterations. Hence, the maximum number of possible distinct sessions is countable. After having described the session length distributions of the synthetic workloads, the high number of distinct sessions is not surprising. Inspecting the structure of the synthetic sessions, we observed the following recurring patterns: (i.) sell inventory,+ (ii.) inven- 


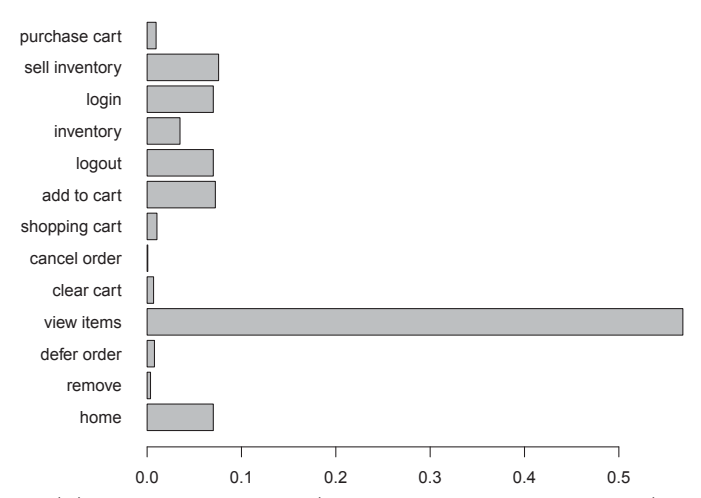

(a) Relative counts (common to all workloads)

\begin{tabular}{r|l||r|r|r|r||r}
\cline { 2 - 6 } & Request & Orig. & ED-2 & NED-3 & NED-4 & Rel. \\
\hline \hline 1 & add to cart & 63,761 & 63,316 & 64,250 & 61,838 & 0.07 \\
\hline 2 & cancel order & 632 & 607 & 634 & 591 & 0.00 \\
\hline 3 & clear cart & 6,047 & 5,941 & 6,140 & 5,843 & 0.01 \\
\hline 4 & defer order & 6,782 & 6,799 & 6,863 & 6,651 & 0.01 \\
\hline 5 & home & 59,934 & 60,957 & 62,054 & 59,971 & 0.07 \\
\hline 6 & inventory & 30,596 & 30,212 & 31,378 & 29,808 & 0.03 \\
\hline 7 & login & 61,500 & 60,957 & 62,054 & 59,971 & 0.07 \\
\hline 8 & logout & 59,934 & 60,957 & 62,054 & 59,971 & 0.07 \\
\hline 9 & purchase cart & 8,360 & 8,328 & 8,351 & 8,139 & 0.01 \\
\hline 10 & remove & 3,027 & 2,993 & 3,044 & 3,064 & 0.00 \\
\hline 11 & sell inventory & 66,679 & 65,413 & 67,691 & 64,794 & 0.08 \\
\hline 12 & shopping cart & 9,074 & 8,934 & 9,184 & 8,907 & 0.01 \\
\hline 13 & view items & 498,601 & 492,675 & 499,983 & 485,611 & 0.57 \\
\hline \hline & $\sum$ & 874,927 & 868,089 & 883,680 & 855,159 & 1.00
\end{tabular}

(b) Absolute and relative counts

Figure 5: Request count statistics

tory + , (iii.) view items + , (iv.) (view items, add to cart) + , (v.) (view items, add to cart, shopping cart, clear cart)+. These patterns can be explained by the corresponding transitions with high probabilities already indicated by the probabilities of the original workload depicted in Figure 3.

Considering the setting for SPECjEnterprise2010, the following conclusions can be drawn about the impact of the clustering results on the session-based metrics session length and number of distinct session types. No statistically significant differences between the synthetic workloads for 2, 3, and 4 clusters can be observed. Both the session length distributions and the number of distinct sessions deviate from the characteristics of the original workload. The deviation of the session length distributions is mainly caused by a number of synthetic long sessions. The mean value shows no statistically significant difference.

\subsubsection{Request Counts}

Figure 5 depicts statistics about the frequency of invoked requests. Based on the absolute numbers of requests to the 13 SPECjEnterprise2010 request types. We computed the relative frequencies for the original workload and the three synthetic workloads. An exact match of the relative frequencies could be observed. That is, the deviation, e.g., in form of the sum of squared errors, is zero. Hence, from the server-perspective, the synthetic workloads provide representative workloads in terms of the distributions of requests. A barplot, which looks the same for each of the four workloads, is shown in Figure 5a.

\section{CONCLUSION AND FUTURE WORK}

In this paper, we presented our WessBas approach for the systematic extraction and specification of probabilistic workloads for session-based systems including a transformation to the load testing tool Apache JMeter. To address the challenge of specifying workloads for different workload tools, we first introduced a domain-specific language that describes the structure of a workload in a generalized way. Additionally, we demonstrated how groups of customers with similar behavioral patterns can be identified using clustering algorithms. Finally, the evaluation with the standard industry benchmark SPECjEnterprise2010 demonstrated the practicality and high accuracy of the proposed approach.

As future work, we plan to further automate the generation of Application Models, including Protocol Layer and test data, as well as automatic learning of guards and actions [12]. We want to extend the set of supported logging formats and load testing tools. The measurement-based approach will be combined with model-based performance evaluation approaches [16] by generating workload specifications of performance models from WESSBAS-DSL instances. Moreover, the evaluation of other clustering algorithms and the integration of approaches for the generation of varying workload intensities [13] will be investigated.

\section{REFERENCES}

[1] P. Barford and M. Crovella. Generating representative web workloads for network and server performance evaluation. In Proc. SIGMETRICS '98, pages 151-160, 1998.

[2] P. Berkhin. A survey of clustering data mining techniques. In Grouping multidimensional data, pages 25-71. Springer, 2006.

[3] A. Brunnert, C. Vögele, A. Danciu, M. Pfaff, M. Mayer, and H. Krcmar. Performance management work. Business $\&$ Information Systems Engineering, 6(3):177-179, 2014.

[4] M. Hall, E. Frank, G. Holmes, B. Pfahringer, P. Reutemann, and I. H. Witten. The WEKA data mining software: An update. ACM SIGKDD Explor. Newsletter, 11(1):10-18, 2009.

[5] R. Jain. The Art of Computer Systems Performance Analysis. John Wiley \& Sons, New York, 1991.

[6] D. Krishnamurthy, J. A. Rolia, and S. Majumdar. A synthetic workload generation technique for stress testing session-based systems. IEEE TSE, 32(11):868-882, 2006.

[7] D. A. Menascé. Load testing of web sites. In IEEE Internet Computing, pages 70-74, 2002.

[8] D. A. Menascé, V. A. F. Almeida, R. Fonseca, and M. A. Mendes. A methodology for workload characterization of e-commerce sites. In Proc. EC '99, pages 119-128, 1999.

[9] D. Pelleg, A. W. Moore, et al. X-means: Extending K-means with efficient estimation of the number of clusters. In ICML '00, pages 727-734, 2000 .

[10] E. Schulz. Integrating performance tests in a generative software development platform, 2014. Master's Thesis, Kiel University, Germany.

[11] E. Schulz, W. Goerigk, W. Hasselbring, A. van Hoorn, and $\mathrm{H}$. Knoche. Model-driven load and performance test engineering in DynaMod. In Proc. MMSM '14, pages 10-11, 2014.

[12] M. Shams, D. Krishnamurthy, and B. Far. A model-based approach for testing the performance of web applications. In Proc. SOQUA 2006, pages 54-61. ACM, 2006.

[13] J. v. Kistowski, N. R. Herbst, and S. Kounev. Modeling variations in load intensity over time. In Proc. $L T$ '14, pages 1-4. ACM, 2014

[14] A. van Hoorn, M. Rohr, and W. Hasselbring. Generating probabilistic and intensity-varying workload for web-based software systems. In Proc. SIPEW' 08 , pages 124-143, 2008.

[15] A. van Hoorn, J. Waller, and W. Hasselbring. Kieker: A framework for application performance monitoring and dynamic software analysis. In Proc. ICPE '12, pages 247-248, 2012.

[16] C. Vögele, A. Brunnert, A. Danciu, D. Tertilt, and H. Krcmar. Using performance models to support load testing in a large SOA environment. In Proc. LT '14, pages 5-6. ACM, 2014.

Acknowledgment: This work has been supported by the Research Group of the Standard Performance Evaluation Corporation (SPEC). 